\title{
ZOOPLÂNCTON DO RIO ITAJAÍ-ACÚ A JUSANTE DA CIDADE DE BLUMENAU, SANTA CATARINA, BRASIL
}

\author{
Zooplankton of Itajai-Açú River, jusant of Blumenau city, \\ Santa Catarina, Brazil
}

\author{
MoacyrSerafim-Júnior ${ }^{l}$ \\ GilmarPerbicheNeves ${ }^{2}$ \\ Lineude Brito ${ }^{3}$ \\ AndréRicardo Ghidini ${ }^{4}$
}

\section{Resumo}

Nesse trabalho foram listadas e caracterizadas as espécies zooplanctônicas coletadas em cinco pontos no rio Itajaí-Acú, a jusante da cidade de Blumenau, Santa Catarina, no mês de julho de 2003. Foram identificadas 23 espécies de Rotifera, 22 de Cladocera e seis de Copepoda. Espacialmente, os pontos com fluxo mais lêntico e fundo arenoso-lodoso registraram maior número de espécies. Foram observadas maior riqueza e densidade de Rotifera, organismos esses considerados cosmopolitas e com um amplo espectro alimentar. Duas famílias de Cladocera, Bosminidae e Chydoridae apresentaram grande diversidade e densidade. A primeira, geralmente herbívora, foi registrada nos pontos com características mais lênticas, e a segunda é tipicamente bentônica, representada por várias espécies em todos os pontos. Copepoda registrou baixa densidade por ser um ambiente lótico, com o domínio das formas larvais (nauplio e copepodito) e maior número de espécies nos pontos mais lênticos. No presente estudo, o rio encontravase em intenso período de estiagem, podendo ser esse o fator da baixa diversidade em geral observada em todos os pontos. Todas as espécies registradas são comuns na região tropical.

Palavras-chave: Zooplâncton; Rotifera; Cladocera; Copepoda; Composição.

Dr. Pontifícia Universidade Católica do Paraná, Laboratório de Ecologia Aquática. e-mail: m.serafim@pucpr.br

Biólogo, Doutorando na Universidade Estadual Paulista - Júlio de Mesquita Filho.e-mail: perbiche@ibb.unesp.br

Biólogo, Mestrando na UFPR, Centro de Estudos do Mar. e-mail: lineubrito@yahoo.com.br

Biólogo, Doutorando no Instituto Nacional de Pesquisas da Amazônia. e-mail: ghidini@inpa.gov.br 


\section{Abstract}

This paper listed and characterized the zooplankton species collected on five stations on the Itajaí-açú river, jusant of Blumenau city, Santa Catarina state, in July 2003. Were identified 23 species of Rotifera, 22 Cladocera and 6 Copepoda. Spatially, the stations with lentic flow and sand-mud deep occurred the largest number of species. Were observed higher richness and density of Rotifera, this organisms considered cosmopolitan and with a large food spectral. Two Cladocera families, Bosminidae and Chydoridae showed high diversity and density, the first generally herbivorous was registered on lentic characteristics stations, and the second is tipically benthonic, represented for many species in all stations. Copepoda registered low density because the environment is lotic, the larval stage (nauplii and copepodit) was dominant and more species occurred in lentic stations. In the present study the river found in intensively drought, which can responsible to low diversity in general observed on all stations. All species registered were common on tropical region.

Keywords: Zooplankton; Rotifera; Cladocera; Composition.

\section{Introdução}

Os rios são sistemas que apresentam, da cabeceira à foz, uma sucessão de valores fisiográficos, físico-químicos e bióticos. São sistemas abertos, de fluxo contínuo, em que os nutrientes recebidos são carreados continuamente para longe do local de liberação e, juntas, essas características influenciam na distribuição espacial da comunidade zooplanctônica (1).

A variação do fluxo é um dos mais importantes fatores que modificam a abundância do zooplâncton ao longo do rio. O fluxo intenso reduz a densidade deles e traz espécies adicionais dos tributários. Em contraste, rios com baixos fluxos desenvolvem comunidades típicas de ecossistemas lênticos (2).

O zooplâncton é um importante elo de energia e massa entre os produtores e os demais consumidores e a sua composição pode servir de ferramenta de avaliação para o estado trófico do sistema, pois a reprodução de diversas espécies é rapidamente afetada em ambientes que recebem influências antrópicas (3).

Na região tropical ocorre a nítida sucessão de espécies características dos períodos secos e chuvosos, representados geralmente pelo inverno e verão. Espécies de rápida reprodução e amplo espectro alimentar predominam em épocas de pouco alimento e homogeneidade na coluna de água. Esses organismos são chamados pelos ecólogos como $r$ estrategistas, o contrário dos $k$-estrategistas, que desenvolvem populações em um ritmo mais lento e quando o meio e a oferta de alimento são favoráveis para sua reprodução (4).
De acordo com essas considerações, o objetivo do presente trabalho foi listar as espécies zooplanctônicas existentes no rio Itajaí-acú e caracterizá-las com o tipo de hábitat coletado.

\section{Materiais e métodos}

O rio Itajaí-açú está localizado na vertente do litoral ou atlântico, formado inicialmente pelos rios Itajaí do Oeste e Itajaí do Sul. Mais adiante, recebe as águas do rio Itajaí do Norte, também chamado de Hercílio, sendo o mais extenso de todos (5). O rio Itajaí do Norte, muitas vezes considerado um dos formadores do Itajaí-Açú, é apenas um dos afluentes da margem esquerda. Os outros afluentes da margem esquerda são: Rafael, Itoupava, Cedros, Testo, Areal e Luiz Alves. Na margem direita: Engano, Trombudo, Garcia, Gaspar Grande, Poço Grande, Gaspar Pequeno e Itajaí-Mirim.

Geologicamente, a região é caracterizada em grande parte pelo Embasamento Cristalino, com a presença de granitos, que configuram geralmente os altos topográficos, representados pelo Morro do Baú (município de Ilhota), onde se encontra o Parque Botânico do Morro do Baú, única área preservada na forma de unidade de conservação na região, e o Morro do Spitzkopf, cujas imediações atingem a porção sul do município de Blumenau (6).

O clima da região pode ser caracterizado como mesotérmico úmido com verões quentes e invernos brandos, $c f a$ segundo a classificação de Köpppen, com temperaturas médias anuais entre $18^{\circ} \mathrm{C}$ e $20^{\circ} \mathrm{C}$ e com pluviosidade variando entre 1600 e 1800 
mm anuais. Associada ao clima $c f a$, encontramos uma vegetação conhecida como Floresta Ombrófila Densa, Floresta Tropical Atlântica, Floresta Latifoliada úmida de Encosta, Floresta Pluvial Costeira ou ainda Mata ou Floresta Atlântica.

No período da coleta do presente trabalho, em julho de 2003, o rio encontrava-se em uma extensa estiagem, considerada como uma das piores das últimas décadas, a qual reduziu o volume de água para $40 \%$ da média normal.

As coletas foram realizadas em cinco pontos determinados conforme a variação morfológica e de hábitat dos rios (Figura 1). Para cada ponto de coleta foram filtrados 300 litros de água da superfície, com auxílio de balde graduado, em rede de plâncton de 64 micrômeros de abertura.
O material resultante foi fixado em solução de formol a 4\%, tamponado com tetraborato de cálcio. As análises das amostras foram feitas no laboratório de Plâncton da PUCPR, Câmpus Curitiba.

Para cada grupo zooplanctônico foram utilizadas bibliografias especializadas, apresentadas na Tabela 1. Os organismos foram observados em câmara de Sedgwick-Rafter, sob microscópio óptico binocular e esteroscópio. Realizaram-se subamostragens com pipeta tipo Stampell de cada amostra, quantificando no mínimo 100 indivíduos de cada grupo.

Os resultados foram tabulados em Microsoft Excel 2000 e a partir da densidade relativa por metro cúbico foram obtidas informações da distribuição espacial nos cinco pontos amostrados.

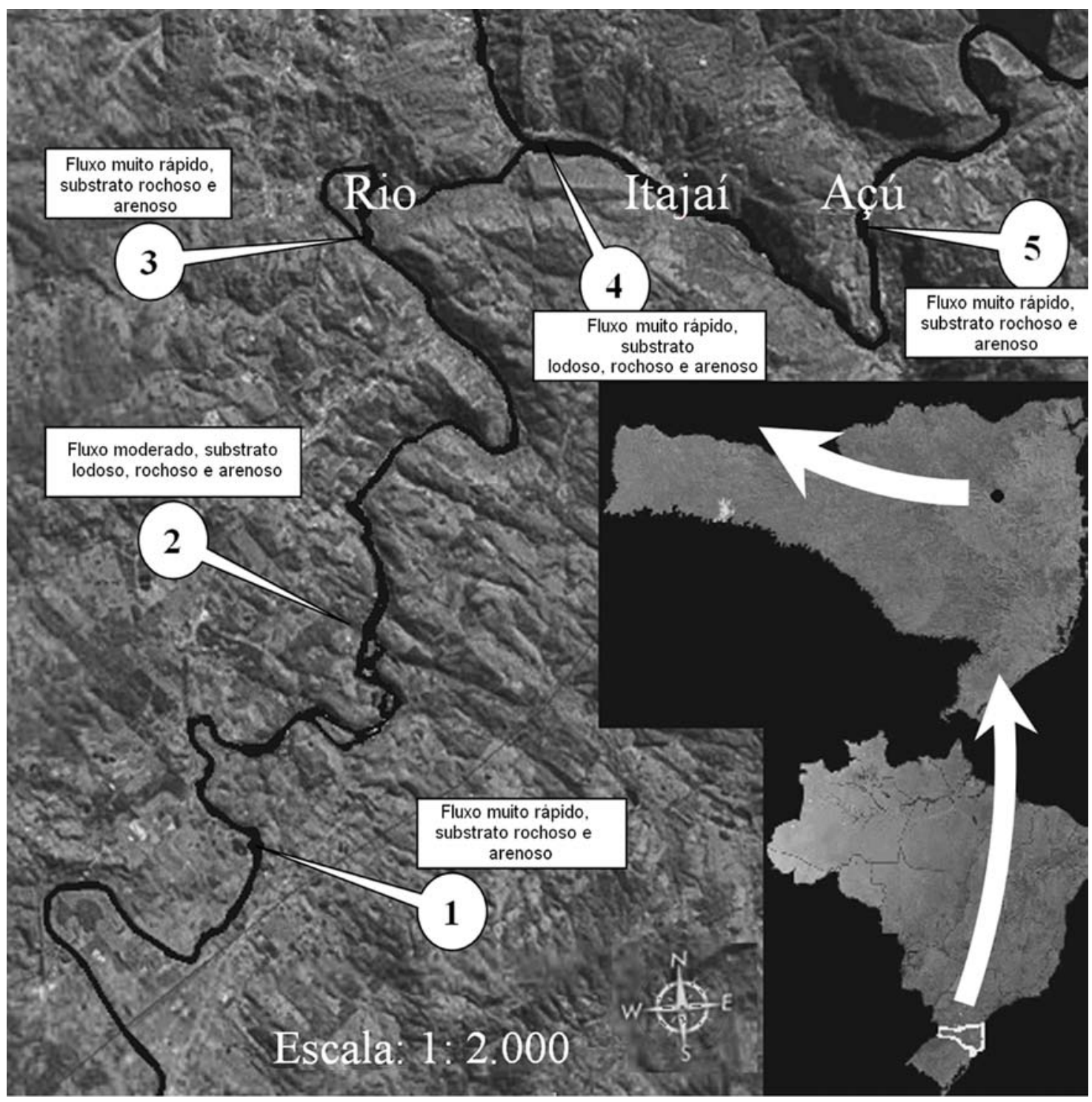

\section{FIGURA 1 - Localização da área de estudo e dos pontos de amostragem (Image 2006 Terra Metrics)}


TABELA 1 - Referências bibliográficas utilizadas para a identificação específica de cada grupo zooplanctônica no Laboratório de Plâncton da PUCPR

\begin{tabular}{lc}
\hline Grupo zooplanctônico & Referências \\
\hline Rotifera & KOSTE (7); SEGERS (8) \\
Cladocera & ALONSO (9); ELMOOR-LOUREIRO (10); KORÍNEK (11); SMIRNOV (12) \\
Copepoda & REID (13)
\end{tabular}

\section{Resultados e discussão}

Nas amostras analisadas, foram identificadas 51 espécies, destas 23 espécies pertencentes aos Rotifera, 22 aos Cladocera e 6 aos Copepoda.

As famílias mais representativas para os Rotifera foram Brachionidae (seis espécies) e Lecanidae (quatro espécies), sendo essas comumente registradas em ambientes aquáticos continentais brasileiros (Tabela 2).

Entre os Cladocera, as famílias Chydoridae (oito espécies) e Bosminidae (cinco espécies) foram as mais representativas (Tabela 2). As espécies pertencentes à família Chydoridae são organismos não planctônicos e as espécies que fazem parte da família Bosminidae são tipicamente planctônicas, ambas são freqüentemente registradas em ambientes de água doce $(16,17,18)$.

Para os Copepoda, apenas as espécies da ordem Cyclopoida (seis espécies) foram encontradas nas amostras (Tabela 2). As espécies da ordem Calanoida não foram registradas, que em ambientes aquáticos lóticos é comum o número reduzido de espécies devido à grande instabilidade do sistema $(18,19)$.

Em rios, as variáveis que definem as condições ecológicas são a longitude do curso, velocidade do fluxo e sua duração; também aplicadas a rios onde o tempo de residência da água, a carga de sólidos em suspensão (orgânico e inorgânico), a extensão e heterogeneidade da altura de inundação se transformam em indicadores dos organismos que eles habitam $(20,21,22)$. Existem controvérsias quanto ao uso do zooplâncton lótico como indicador do ambiente, onde alguns autores como Bonecker e Lansac-Tôha (23) consideram que a descarga de restos líquidos, mesmo alterando algumas propriedades limnológicas da água, não influencia na riqueza da comunidade, enquanto que Marneffe et al. (3) cita que o zooplâncton responde rapidamente à qualidade da água no meio e às condições ambientais.
Os rotifera registraram maior número de espécies e densidade de organismos, na maioria dos pontos (Figura 2). Estes constituem o grupo dominante de zooplâncton da maioria das águas continentais, representando mais de $60 \%$ do total dessa comunidade. Por serem organismos oportunistas, adaptam-se com facilidade às mudanças das condições ambientais e, assim, dificilmente se encontra um padrão constante de composição e ocorrência de espécies no espaço e no tempo (24). Sabri (25) e Petts (26) afirmam que a densidade de população de Rotifera decresce rapidamente rios abaixo comparados a outros grupos zooplanctônicos. Evans (27) referiu que as distribuições espaciais e temporais das espécies de Rotifera não são ao acaso e sugeriu que há uma evidência de competição explorativa entre os táxons, favorecendo os mais adaptados às variações do sistema, mesmo que os recursos disputados não estejam necessariamente em falta, havendo uma alternância da abundância nos pontos estudados.

O zooplâncton de pequeno porte pode aparecer em densidades maiores do que outros grupos zooplanctônicos e crustáceos em águas naturais, como o ocorrido do rio Itajaí (2) (Figura 3).

A alta diversidade de Cladocera registrada no presente estudo, representada na Tabela 3 e Figura 2, esteve composta com maiores valores pelas famílias Chydoridae e Bosminidae. A alta diversidade da família Chydoridae é padrão em sistemas lóticos, por estarem mais bem adaptadas ao tipo de alimentação detritivora, utilizando as regiões bentônicas para proteção contra predadores e reprodução. Também, existe a deriva de espécies tipicamente planctônicas de locais lênticos, como planícies e lagoas marginais, além de pequenos reservatórios. Muitos destes organismos também são freqüentemente associados à região litorânea de reservatórios, além de associados a bancos de macrófitas (28). 
TABELA 2 - Lista de espécies, ocorrência e freqüência entre as amostras de Rotifera, Cladocera e Copepoda registrados no rio Itajaí

Famílias e Espécies

Pontos

1

2

Rotifera

Collothecidae

Collotheca sp.

Conochilidae

Conochilus coenobasis

Conochilus unicornis

Floscularidae

Ptygura sp.

Brachionidae

Brachionus mirus var. reductus

Kellicottia bostoniensis

Keratella cochlearis

Keratella lenzi

Keratella tropica

Platyias quadricornis quadricornis

Colurellidae

Lepadella tenella

Euchlanidae

Euchlanis meneta

Gatropodidae

Ascomorpha saltans

Lecanidae

Lecane bulla

Lecane cornuta

Lecane hornemanni

Lecane lunaris

Mytilinidae

Mytilina ventralis

Notommatidae

Cephalodella gibba

Proalidae

Proales sp.

Synchaetidae

Polyarthra vulgaris

Trichocercidae

Trichocerca pusilla

Trichotriidae

Trichotria tetractis tetractis

Ordem Bdelloidea

Cladocera

Bosminidae

Bosmina hagmanni

Bosmina buaronensis

Bosmina longirostris

Bosmina tubicen

Bosminopsis deitersi

$\begin{array}{lll}X & X & \\ & & \\ X & X & X \\ X & X & X\end{array}$

X

X

$\mathrm{X}$

80

X

20

X

$X$

20

X

X

$\mathrm{X}$

X

X

X

$\mathrm{X}$

80

20

20

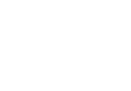

$\mathrm{X}$

X X X

60

X

X

X

60

X

$$
\text { X }
$$

X

X

$\mathrm{X}$

$\mathrm{X}$

$\mathrm{X}$

$\mathrm{X}$

X

80

60

20

20

$\mathrm{X}$

$\mathrm{X}$

60

$\mathrm{X}$

$\mathrm{X}$

60

X

$\mathrm{X}$

X

$\mathrm{X} \quad \mathrm{X}$

X

X

$\mathrm{X}$

X 
Famílias e Espécies

\section{Chydoridae}

Alona guttata

Alona intermedia

Alona monacantha

Alona sp.

Chydorus eurynotus

Chydorus nitidulus

Disparalona dadayi

Leydigiopsis brevirostris

Notoalona sp.

Daphniidae

Ceriodaphnia reticulata

Ceriodaphnia silvestrii

Daphnia gessneri

Scapholeberis sp.

Ilyocryptidae

Ilyocryptus spinifer

Macrothricidae

Macrothrix mira

Macrothrix cf. paulensis

Moinidae

Moina micrura

\section{Copepoda}

\section{Cyclopidae}

Eucyclops cf. ensifer

Mesocyclops meridianus

Microcyclops anceps anceps

Microcyclops ceibaensis

Paracyclops chiltoni chiltoni

Tropocyclops prasinus prasinus

Harpacticoida

$\mathrm{X}$
$\mathrm{X}$
$\mathrm{X}$
$\mathrm{X}$
$\mathrm{X}$

X

X

$\mathrm{X}$

$\mathrm{X}$

$\mathrm{X}$

X

X $\quad \mathrm{X}$

X

X

40

$\mathrm{X}$

$X$
$X$

X

100

$\mathrm{X}$

$\mathrm{X}$

100

20

40

20

60

20

40

60

$\mathrm{X}$
$\mathrm{X}$

X

40

X $\quad \mathrm{X}$

60

X

$\begin{array}{ll}X & X \\ X & X\end{array}$

40

X
X

$\begin{array}{ll} & \text { X } \\ X & \text { X } \\ \text { X } & \text { X } \\ \text { X } & \\ \text { X } & \text { X } \\ \text { X } & \end{array}$

20

20

80

40

20

40

60

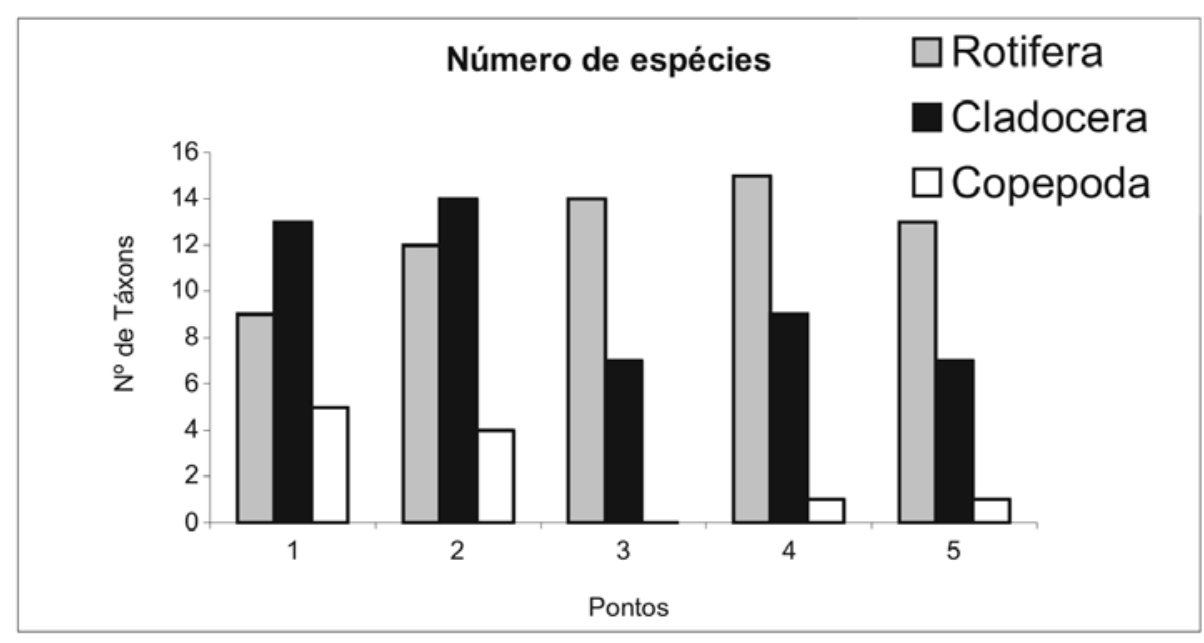

FIGURA 2- Número de espécies de Rotifera, Cladocera e Copepoda registrados no presente estudo 
A escassa abundância dos Cladóceros registrada no presente estudo associa-se à presença de sólidos em suspensão e não aos peixes planctívoros, cuja fonte principal de alimento é o plâncton adventício $(29,30)$ (Figura 3B).

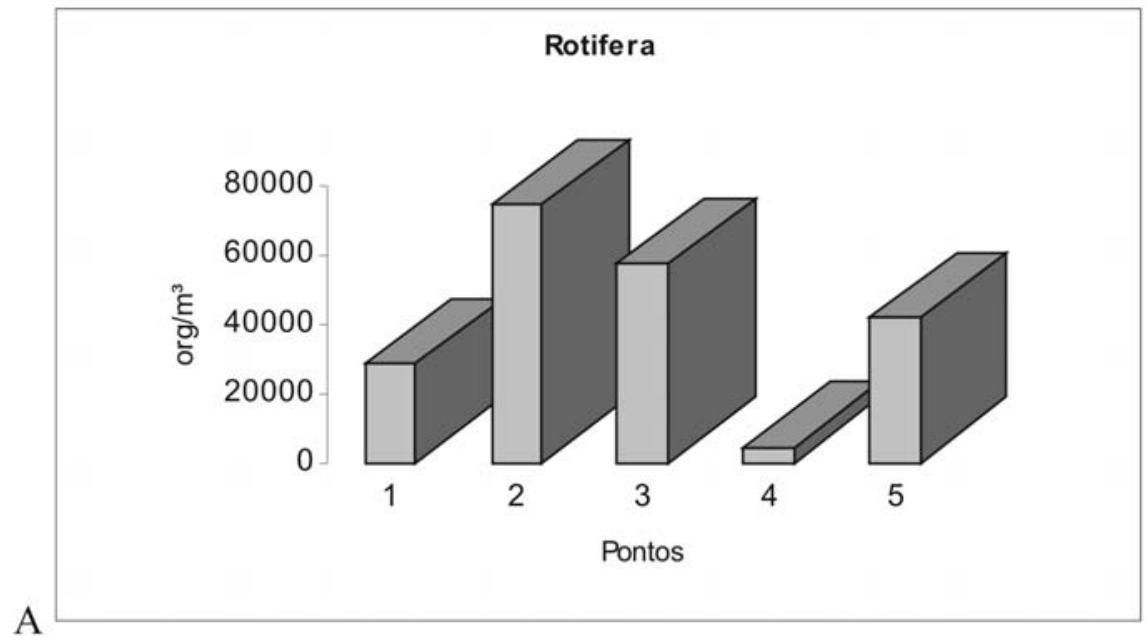

A

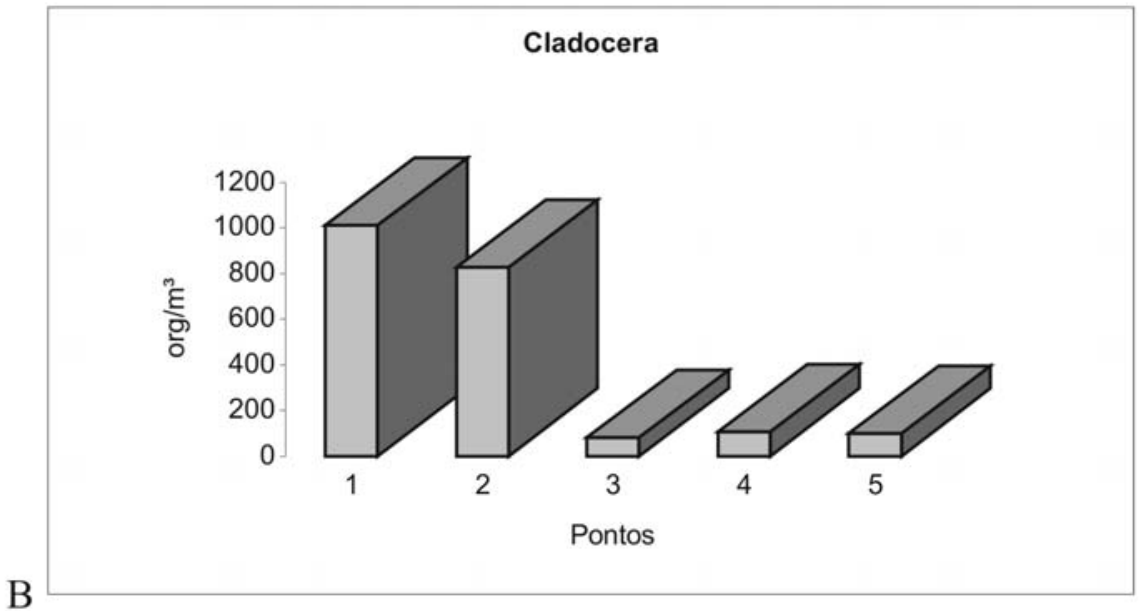

$\mathrm{B}$

\section{Copepoda Cyclopoida}

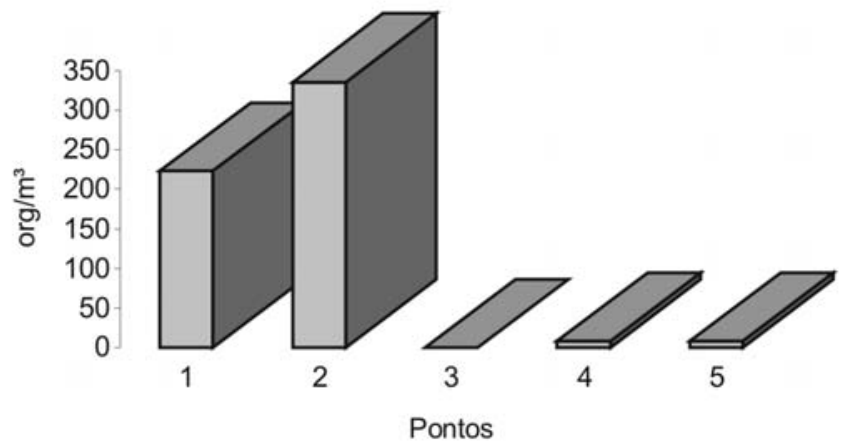

$\mathrm{C}$

FIGURA 3 -Distribuição espacial (organismos por metro cúbico) nos pontos amostrados. A. Rotifera. B. Cladocera. C. Copepoda Cyclopoida. D. Copepoditos de Cyclopoida e Calanoida. E. Náuplios de Cyclopoida 
Bosminopsis deitersi, Bosmina hagmanni, Moina minuta e Ceriodaphinia cornuta são encontradas em grande abundância no rio Paraná, na Argentina (20), no baixo rio Paraná $(31,32)$, no alto rio Paraná $(33)$, na região Amazônica $(34,14)$ e em planícies de inundação da Venezuela $(35,36)$, e ainda podem ser considerados de vasta ocorrência na América do Sul, Estados Unidos, África e Região Oriental do Sudeste da Ásia (37).

As características hidrológicas destes cursos de água não proporcionam aos Cladocera locais ideais de desenvolvimento, tendo em vista que as baixas transparências causadas pela grande quantidade de material em suspensão promovem a escassez de alimento para estes organismos, o fitoplâncton (38).

Outro fator que também possui influência nas populações de Cladóceros é a velocidade da água. Organismos mais eficientes na natação conseguem desenvolver-se nestes ambientes. Já organismos mais lentos são carregados pela correnteza. Neste caso, áreas de remanso e bancos de macrófitas representam locais de refúgio para estes organismos (39).

Os Copepoda registraram baixos valores de número de espécies e de densidade, com maiores valores nos pontos 1 e 2 (Figuras 2 e 3 C, D, E). Formas adultas de Copepoda possuem poucos registros em rios, sendo, porém, muito numerosas as larvas (Nauplio e Copepodito) (29).

A ordem Calanoida tolera diferenças de iluminação no meio ambiente, mas fatores como temperatura, $\mathrm{pH}$ e condutividade são suficientes probabilidades de ocorrer espécies isoladas (40). Além desses fatores, grande número de espécies é herbívora, quando a produção primária nos rios é reduzida comparada aos reservatórios.

$\mathrm{Na}$ ordem Cyclopoida, grande parte das espécies é onívora, tendo disponíveis mais recursos comparados aos Calanoida. Todos os gêneros registrados nesse trabalho são freqüentes em sistemas lóticos $(18,19)$.

\section{Conclusão}

As espécies registradas no presente trabalho são comuns na região tropical e em sistemas lóticos. Os pontos $1 \mathrm{e} 2$ registraram maiores densidades devido às suas características mais lênticas comparadas aos pontos 3,4 e 5, principalmente para os microcustáceos (Cladocera e Copepoda), onde existe maior produção primária. Já estes últimos pontos favoreceram a maior diversidade de Rotifera possivelmente pela maior variedade de hábitats.

\section{Referências}

1. Arrojo P, Gracia JJ. Los trasvases del Ebro a debate. Barcelona: Nueva Cultura del Agua; 2000.

2. Kobayashi T, Shiel R, Gibbs P, Dixon P. Freshwater zooplankton in the Hawkesbury-Nepean River: comparison of community structure with other rivers. Hydrobiologia. 1998; 377(1):133-145.

3. Marneffe Y, Descy JP, Thome JP. The Zooplankton of the louter river Meuse, Belgum: seasonal changes and impact of industrial and municipal discharges. Hydrobiologia. 1996; 319:1-13.

4. Pinto-Coelho RM. Fundamentos de Ecologia. Porto Alegre: Artes Médicas Sul; 2000.

5. Cabral OR. Santa Catarina: história-evolução. São Paulo: Companhia Nacional; 1937.

6. Santa Catarina. Gaplan/Suegi - Atlas de Santa Catarina. Rio de Janeiro: Aerofoto Cruzeiro; 1991.

7. Koste W. Rotatoria. Stuttgart: Gebrüder Bosntraeget; 1978.

8. Segers H. Guides to the identification of the Microinvertebrates of the Continental Waters of the World: Rotifera, The Lecanidae (Monogononta). Spp Academic Publishing; 1995. v. 2.

9. Alonso M. Crustacea, Branchiopoda. In: Ramos MA. Editor. Fauna Ibérica. Madrid: Museo Nacional de Ciencias Naturales, CSIC; 1996. v. 7.

10. El Moor-Loureiro LMA. Manual de Identificação de Cladóceros Límnicos do Brasil. Taguatinga: Universa; 1997.

11. Korínek V. Revision of three species of the genus Diaphanossoma Fischer, 1850. Hydrobiologia. 1987; 145:35-45.

12. Smirnov NN. Cladocera: the Chydorinae and Sayciinae (Chydoridae) of the World. In: Dumont HJF. editor. Guides to the Identification of the Microinvertebrates of the Continental Waters of the World. Amsterdam: SPB Academic Publishing; 1996.

13. Reid JW. Chave de identificação e lista de referencias bibliográficas para as espécies continentais sulamericanas de vida livre da ordem Cyclopoida (Crustacea Copepoda). Boletim de Zoologia. Sao Paulo: 1995; 9:17-143. 
14. Bozelli RL. Composition of the zooplankton of Batata and Massuará lakes and of the Trombeta River. Amazoniana. 1992; 12:239-261.

15. Bonecker CC, Lansac-Toha FA, Staub A. Qualitative study of rotifers in different environments of the high Paraná river floodplain (MS), Brazil. Revista Unimar. 1994; 3:1-16.

16. Mussara ML, Sendacz S, Beyruth Z, Novelli JL. Caracterização Limnológica de Ambientes Lóticos e Lênticos na Área de Influência do Complexo Prof. Maurício Joppert, Bataguassu, MS. Oecologia Brasiliensis. 1995; 2:105-115.

17. Lima AF, Lansac-Toha F, Bonecker CC. Zooplanckton in the floodplains of a tributary to the Paraná River in Mato Grosso do Sul, Brazil. Studies Neotropical Fauna Environmental. 1996; 31:112-116.

18. Lopes RM, Lansac-Toha F, Vale R, Serafim-Júnior M. Comunidade zooplanctônica do reservatório de Segredo. In: Agostinho, AA; Gomes LC. editor. Reservatório de Segredo. Maringá: EDUEM; 1997. p. 39- 60.

19. Lansac-Toha FA, Bonecker CC, Velho LFM, Lima AF. Comunidade zooplanctônica. In: Vazzoler AEAM, Agostinho AA, Hahn NS. editor. A planície de inundação do alto Rio Paraná: aspectos físicos, biológicos e socioeconômicos. Maringá: EDUEM; 1997. p. 115-153.

20. Rzoska J. The Nile: Biology of an Ancient river, Monographes Biological, 29 W. Junk, The Hague; 1976.

21. Neiff JJ. Ideas para la interpretación ecológica del Paraná. Interciencia. 1990; 15(6):424-441.

22. Neiff JJ. Aspectos conceptuales para la evaluatión ambiental de tierras humedas continentales de America del Sur. Anais Semin. Region. Ecol. 1996; 8(1):1-19.

23. Bonecker CC, Lansac-Toha FA. Community structure of rotifers in two environments of the river Paraná floodplain (MS), Brazil. Hydrobiologia. 1990; 325(2):137-150.

24. Rodriguez MP, Matsumura-Tundisi T. Variation of density, species composition and dominance of Rotifers at a shallow tropical reservoir (Broa reservoir, SP, Brazil) in a short scale time. Revista Brasileira de Biologia. 2000; 60:1-7.
25. Sabri AW. Ecological studies on Rotifera (Aschelminthes) in the river Tigris, Iraq. Acta Hydrobiol. 1988; 30:367-379.

26. Petts GE. Impounded river-perspectives for ecological management. Wiley: Chichester; 1984

27. Evans WA. Seasonal abundances of the psammic rotifers of a physically controlled stream. Hydrobiologia. 1984; 108:105-114.

28. Paggi JC, José de Paggi S. Zooplankton de ambientes lóticos e lênticas do rio Paraná médio. Acta Limnol. Brasil. 1990; 3:685-719.

29. José de Paggi S. Estudios limnológicos en una sección transversal del tramo medio del río Paraná. X: Distribución estacional del zooplancton. Rev. Asoc Cienc Nat Litoral. 1984; 15(2):135-155.

30. Hardy ER. Changes in species composition of Cladocera and food avaibility in a floodplain lake, Lago Jacaretinga, Central Amazon. Amazoniana. 1992; 12(2):155-168.

31. Lansac-Toha FA, Lima AF, Thomaz SM, Roberto MC. Zooplâncton de uma planície de inundação do Rio Paraná. I. analise qualitativa e estrutura da comunidade. Revista Unimar. 1992; 14:39-60.

32. Lansac-Toha FA, Lima AF, Thomaz SM, Roberto MC. Zooplâncton de uma planície de inundação do Rio Paraná, II. Variação sazonal e influencia dos níveis fluviométricos sobre a comunidade. Acta Limnol Bras. 1993; 6:42-55.

33. Sendacz S. A study of the zooplankton community of Billings reservoir São Paulo, Hydrobiologia. 1984; 133:121-127.

34. Robertson B, Hardy ER. Zooplankton of Amazonian lakes and rivers. In: Sioli H. The Amazon: Limnology and landscape ecology of a mighty tropical river and its basin. The Hague, Junk WJ. 1984; p. 337-352.

35. Vásquez E, Rey J. A longitudional study of zooplankton along the lower Orinoco River and its Delta (Venezuela). Annals Limnol. 1989; 28:3-18.

36. Hamilton SK, Sippel SJ, Lewis WM, Saunders JF. Zooplankton abundance and evidence for its reduction by macrophyte mats in two Orinoco floodplain lakes. Journal of Plankton Research. 1990; 12:345-363. 
37. Weibezahn FH, Vásquez E, Roa EZ, Rey J. Bosminopsis negrensis Brandorff, 1976 (Cladocera, Bosminidae) em La Cuenca de Rio Orinoco al Sur de Venezuela. Memoria Sociedad de Ciências Naturales La Salles. 1990; 45-54.

38. Ringelberg J. Introductory remarks: causal and teleological aspects of diurnal vertical migration. In: Kerfoot WC. editor. Evolution and ecology of Zooplankton communities. Special symposium. Vol. 3. American Society of Limnology and Oceanography. University press of New England. Hanover, New Hampshire; 1980. p. 65-68.
39. Larsson P, Kleiven OT. Food search and swimming speed in Daphnia. In: Lenz PH. Zooplankton: Sensory Ecology and Physiology. Amsterdam: Gordon and Breach Publishers; 1996. p. 375-387.

40. Matsumura-Tundisi T. Latitudinal Distribution of Calanoida Copepods in Freshwater aquatic Systems of Brasil. Revista Brasileira de Biologia. 1986; 46(3):527-553.

Recebido em/Receive din: June 06, 2006 Aceito em/Accepted in: September 12, 2006 